\title{
Are there constraints on learned responses to odors from rewarded and nonrewarded rats?
}

\author{
PAUL J. ESLINGER and H. WAYNE LUDVIGSON \\ Chemistry of Behavior Program, Texas Christian University, Fort Worth, Texas 76129
}

\begin{abstract}
This study determined if test rats could utilize biological odors, generated from donor rats receiving reward $(\mathrm{R})$ and frustrative nonreward $(\mathrm{N})$ treatments, to predict reward and nonreward goal events equally well. In Phase 1, two groups of test rats were exposed to $\mathrm{R}$ and $\mathrm{N}$ odors that signaled, respectively, either $R$ and $N$ goal events ("same" condition) or $N$ and $R$ goal events ("opposite" condition). Rats demonstrated significant discriminative use of these odors under both conditions. Subjects in the "opposite" condition, however, were slightly slower to learn the discrimination. Reversal learning was readily accomplished in Phase 2, regardless of the same-opposite factor. Thus, little evidence for a constraint on learning was found, and an interpretation in terms of interfering response tendencies and their habituation seemed favored.
\end{abstract}

It has been proposed that rats experiencing treatments of reward (R) and frustrative nonreward $(\mathrm{N})$, that is, nonreward in the presence of cues previously associated with reward, excrete differential odors that conspecifics can perceive and utilize (e.g., Ludvigson \& Sytsma, 1967; Prytula \& Davis, 1976; Seago, Ludvigson, \& Remley, 1970; Eslinger \& Ludvigson, Note 1). The R and $\mathrm{N}$ odor excretions appear to elicit unlearned approach and avoidance tendencies, respectively, and faster escape from $\mathrm{N}$ than from $\mathrm{R}$ odor (Collerain, 1978; Collerain \& Ludvigson, 1972, 1977; Mellgren, Fouts, \& Martin, 1973). Furthermore, $R$ and $N$ odors can serve as discriminative cues for corresponding goal events of reward and nonreward (e.g., Ludvigson, 1969; Taylor \& Ludvigson, Note 2).

An intriguing question that arises from these studies concerns the interaction of the unlearned approach and avoidance reactions with the acquisition of learned approach responses. Several studies have demonstrated that unlearned avoidance reactions to $\mathrm{N}$ odor usually habituate over trials (Collerain \& Ludvigson, 1972, 1977); however, with extended experience, some rats seem to develop a stable avoidance response (Ludvigson, McNeese, \& Collerain, 1979). With regard to $\mathrm{R}$ odor, data are few, but the approach reactions may not habituate when the odor is generated by partially rewarded donors (Collerain \& Ludvigson, 1977).

Given these results, one might expect some initial interference with learning when unlearned reactions are

This article is based upon research submitted by the first author to Texas Christian University in partial fulfillment of the Master of Science degree in psychology. The work received partial support from the Texas Christian University Research Foundation and the Chemistry of Behavior Program. Reprint requests should be addressed to H. W. Ludvigson, Department of Psychology, Chemistry of Behavior Program, Texas Christian University, Fort Worth, Texas 76129. "opposite" to the learned tendencies to be established by reinforcement contingencies, but to the extent the unlearned reactions habituate, the interference might be short-lived. Since most pertinent conditioning studies have required the acquisition of instrumental responses that are similar to the unlearned reactions, this expectation remains untested. For the same reason, it is not clear whether there exists any innate limitation or constraint on the ability of rats to establish associations with these odors, as has been suggested for other biologically significant stimuli (Bolles, 1970; Hinde \& Stevenson-Hinde, 1973; Seligman \& Hager, 1972). Since $\mathrm{R}$ and $\mathrm{N}$ odor productions appear to be common across male and female rats (Eslinger \& Ludvigson, Note 1 ), it is possible that selective pressures have favored the development of this kind of olfactory communication for specific circumstances and specific reactions.

Studies that have some bearing on these matters are few and inconclusive. Morrison and Ludvigson (1970) demonstrated that rats can utilize $\mathrm{R}$ and $\mathrm{N}$ odors as signals for turning left or right in a T-maze in order to obtain food. Thus, these odors would appear to possess some degree of flexibility in their signaling capacities. Using a straight-runway apparatus, Prytula and Davis $(1974,1976)$ found that test rats did not respond differentially to $\mathrm{R}$ and $\mathrm{N}$ odor cues in the startbox when the opposite odor cue and goal event were presented in the goalbox. That is, discriminative responding in start speeds occurred only when the donors' and test subjects' goal events were the same and, thus, only when odor cues in both loci were the same, that is, R-R or N-N. Since the test subjects themselves were also generating discriminative odor cues that were accumulating in the goalbox and controlling differential responding there, it is not surprising that the use of "opposite" donor cues in the startbox produced nondifferential responding there. Assuming that the donor- and test-subject odor cues 
were the same or similar, as suggested by Eslinger and Ludvigson (Note 1), the test subjects were presented with a conditional discrimination (e.g., $\mathrm{N}$ odor in the startbox and $\mathrm{R}$ odor in the goalbox both signaled reward) and a fairly limited number of trials $(40)$ in which to master it. A similar analysis might be applied when the locus of donor odor cues is changed to the run section of the alleyway. With such a procedure, Prytula and Davis (1976) found the same results as with startbox donor cues. Alternatively, it might be argued that rats may not be able to utilize "opposite" odor cues to predict reward contingencies, for example, $\mathrm{N}$ odor signaling reward. However, given the difficulty of conditional discrimination problems plus certain other factors operating in these studies, it would seem that they offer no decisive statement about constraints on learning.

The present study was designed to assess three questions: (1) Can test rats learn to utilize $\mathrm{R}$ and $\mathrm{N}$ odor cues in order to discriminate opposite goal events, that is, $\mathrm{R}$ odor signaling $N$ and $N$ odor signaling $R ;(2)$ is there a difference in the initial development of discrimination between groups receiving the same or opposite schedule of reward as their donors; and, probing the utilization of $\mathrm{R}$ and $\mathrm{N}$ odors further, (3) will discrimination reversal be affected by the same-opposite factor? Based on previous studies, we expected that unlearned reactions to $R$ and $\mathrm{N}$ odors might have some differential effect on the initial development of discrimination. However, since these unlearned reactions have been shown to habituate, their influence in this situation might be only transitory.

\section{METHOD}

\section{Subjects}

The test subjects were 12 naive male albino rats purchased from the Holtzman Company, Madison, Wisconsin, and approximately 110 days of age when the food-deprivation regimen was imposed. Donor subjects were 12 runway-experienced male albino rats, also purchased from the Holtzman Company, that were approximately 150 days of age and had previously been exposed to a double-alternation schedule of $\mathrm{R}$ and $\mathrm{N}$ in the same apparatus used in this experiment. All subjects were individually hou sed in stainless steel cages in a constantly illuminated vivarium.

\section{Apparatus}

The apparatus was a $2.13-\mathrm{m}$ alleyway, $8 \mathrm{~cm}$ wide and $9 \mathrm{~cm}$ high, divided into start, run, and goal sections measuring .3, 1.23 , and $.6 \mathrm{~m}$ in length, respectively. The runway was constructed of wood, painted white, and covered with hinged Plexiglas. A microswitch activated by raising the startbox door and photobeams located $.5, .75,1.36$, and $1.66 \mathrm{~m}$ from the startbox door permitted the recording of four traversal times from start, Run 1, Run 2, and goal sections. A goalbox door prevented retracing into the runway. A round glass food cup, $6.4 \mathrm{~cm}$ in diameter, was present in the goalbox on each trial. Test subjects received their reward from a pellet dispenser that delivered 45-mg Noyes food pellets; donors received their reward from pellets previously placed into the goal section. Stainless steel runway inserts, employed for the purpose of controlling accumulations of animal odor, were constructed to fit snugly into the goalbox, covering the floor and both walls. Holes were cut into the sides of the insert to allow passage of photobeams. White paper covered the entire floor of the alleyway, including the area over the insert. A vacuum cleaner attached to the end of the goalbox was used to exhaust air, and vents in the startbox served as air-entrance portals.

\section{Procedure}

Test subjects were allowed to adjust to vivarium conditions for 7 days after arrival from the supplier. During the following 5 days, donor and test subjects were given reduced food rations until $85 \%$ of ad-lib body weight was obtained. Thereafter, all subjects were maintained at this level. Test animals were randomly assigned to two groups: (1) Group S-O, which received the same schedule (S) as donors in Phase 1 (i.e., R-R, N-N) and a schedule opposite (O) to donors in Phase 2 (i.e., R-N, N-R), or (2) Group O-S, which received the opposite schedule and then the same schedule as their donors in Phases 1 and 2, respectively.

Pilot studies with this procedure demonstrated that the use of two donors for each test subject provided a clear and reliable stimulus cue. Therefore, triplets were established, consisting of two donor rats that provided odor cues for one test rat. These triplets were maintained throughout all testing, with the order of donor placements within each triplet remaining the same. Three test rats from each experimental group, along with their donors, were run in each of two replications. The three pairs of donors that served a particular experimental group (i.e., S-O or O-S) were switched in the second replication so as to serve the other experimental group. Thus, across the two replications, the six different donor pairs that served Group S-O also served Group O-S.

Habituation treatments occurred over 2 days and consisted of (1) $5 \mathrm{~min}$ of free exploration daily in the unbaited runway for test subjects, followed later by $1845-\mathrm{mg}$ pellets in the home cage, and (2) eight daily goalbox placements on a doublealternation schedule of $\mathrm{R}$ and $\mathrm{N}$ for each donor. An $\mathrm{R}$ trial for donors consisted of $45 \mathrm{sec}$ of access to $1845-\mathrm{mg}$ pellets in the food cup located in the middle of the goalbox, and an $\mathrm{N}$ trial was $45 \mathrm{sec}$ of retention in the goalbox with an empty food cup present. These same $\mathrm{R}$ and $\mathrm{N}$ events were utilized throughout the study, except that, for a test subject on $\mathrm{R}$ trials, pellets were dispensed into the food cup located at the rear of the goalbox after the subject had broken the last photobeam.

Beginning with acquisition, all subjects were given 2 days of a double-alternation schedule of reward and nonreward (RRNNRRNN), followed by 1 day of a reversed schedule (NNRRNNRR). On Day 4, members of each experimental group diverged in their schedules such that one half remained on the NNRRNNRR schedule for this day and then continued throughout the rest of the phase with a reversed schedule every 2 days (NNRRNNRR to RRNNRRNN). The other half of the donortest triplets were switched to an RRNNRRNN schedule on Day 4 and then continued with the schedule reversing every 2 days. This procedure was employed to insure that there were mixed goal events (i.e., both $\mathrm{R}$ and $\mathrm{N}$ ) occurring on each of the eight daily trials. Thus, with running order of donor-test triplets randomly determined each day, any residual accumulation of odor would not be predictive of the goal event. All subjects were given Trial 1 before any subject received Trial 2 , and so on.

On a given trial, the first member of a donor pair was brought from a waiting room into the apparatus room, where it was placed directly into the goalbox to receive either $R$ or $N$. Then, the donor was removed to a third room. The same procedure was also followed for the second donor. Next, the appropriate test subject was brought from the waiting room and allowed to traverse the runway. Following each donor-test triplet, the alleyway paper was changed, the metal insert was replaced by another clean insert, and the alley air was exhausted for $90 \mathrm{sec}$. The maximum time a test subject was permitted to remain in the start, Run 1, or Run 2 section of the runway before it entered the goalbox was $60 \mathrm{sec}$; after this time, the animal was gently nudged into the next section.

With this general procedure, the experiment was divided into two phases of 11 days each. In Phase 1 , the double-alternation 
schedule of $\mathrm{R}$ and $\mathrm{N}$ was the same for donor and test rats of Group S-O and the opposite for donor and test rats in Group O-S. After a 1-day break, Phase 2 commenced, with Group S-O switched to the opposite schedule and Group O-S switched to the same schedule as donor subjects.

\section{RESULTS}

Traversal times for each runway measure were converted to speeds in meters per second. A groups (S-O vs. $\mathrm{O}-\mathrm{S}$ ) by goal events ( $\mathrm{R}$ vs. N) by days analysis of variance with repeated measures on goal events and days was employed to analyze these measures over the last 3 days of each phase.

\section{Terminal Discrimination}

Phase 1. Test subjects operating under schedules the same as or opposite to those of their donors discriminated $\mathrm{R}$ and $\mathrm{N}$ goal events, running fast in the presence of odor signaling reward and slowly in the presence of odor signaling nonreward (see Figure 1). Analysis of goalbox speeds revealed a significant effect of goal events $[F(1,10)=41.00, p<.001]$, with no significant interaction with groups or groups and days. Thus, by the end of Phase 1 , animals were able to use $\mathrm{R}$ and $\mathrm{N}$ odors as signals for goal events that were either the same as or opposite to treatments given to donors. Analysis of data from the Run 2 section of the alleyway revealed a significant discrimination $[\mathrm{F}(1,10)=14.38, \mathrm{p}<.01]$ that was slower to develop and smaller in magnitude than goalbox discrimination. No evidence of differential responding appeared in Run 1 speeds or start speeds.

Phase 2. Operating in Phase 2 under reversed schedules from Phase 1, Groups S-O and O-S reversed their discriminative performance. Over the last 3 days in the goal section, both groups ran faster to odor signaling reward that had previously signaled nonreward and slower to odor signaling nonreward that had previously signaled reward $[F(1,10)=14.78, p<.01]$. No other

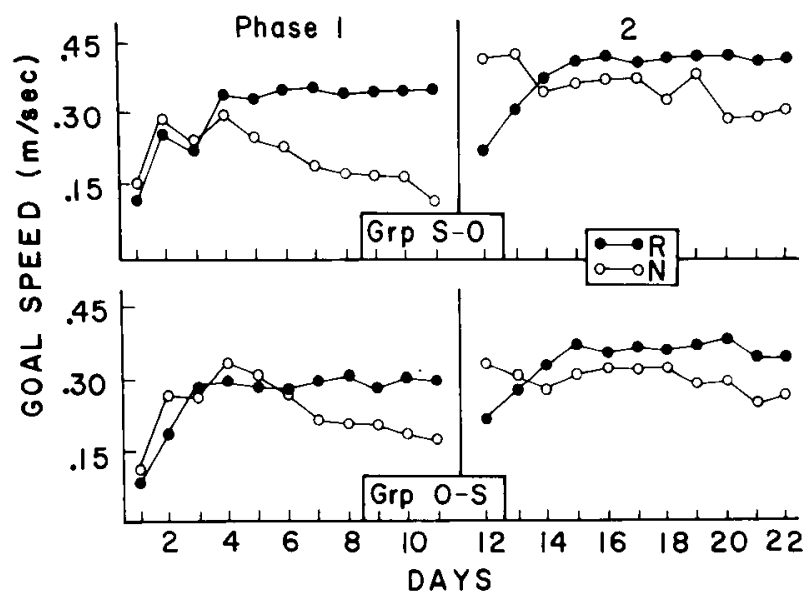

Figure 1. Daily mean goalbox speeds in Phases 1 and 2 for Groups S-O and O-S. main effects or interactions were found to be significant in this measure. Analysis of Run 2 and Run 1 speeds revealed no effects attributable to groups, goal events, or days. Analysis of start speeds revealed that Group S-O ran faster overall than Group O-S $[F(1,10)=6.05$, $p<.05]$. A similar difference was suggested in most other measures, although these were not significant. It appeared that Group S-O was generally somewhat faster than Group O-S, regardless of the experimental manipulation.

\section{Development of Discrimination}

Inspection of Figure 1 suggests that the development of discrimination in Phase 1 was somewhat slower for Group O-S than for Group S-O. Since there was the a priori expectation, based on prior data, of some initial interference with discrimination in Group O-S relative to Group S-O, a trials-to-discrimination analysis designed specifically to evaluate this expectation was performed. Furthermore, to be certain the analysis was independent of any arbitrarily selected discrimination criterion, a graded series of criteria was employed. Based upon the average magnitude of discrimination ( $\mathrm{R}$ speed minus $\mathrm{N}$ speed) across all subjects on the last day of the phase, onset of discrimination was defined as achievement of a given percentage of this average terminal magnitude. The following percentages were used, which then specified a series of criteria for achievement of discrimination onset: $5 \%, 10 \%, 20 \%, 30 \%, 40 \%$, and 50\%. The dependent measure was the ordinal number of the day on which a subject's mean $\mathrm{R}$ trial speed was faster than its mean $\mathrm{N}$ speed by an amount large enough to exceed the specified criterion.

Group means calculated from these dependent measures are presented in Table 1. Differences between groups were analyzed at each percentage criterion by means of a $t$ test. In Phase 1 , significant differences appeared between groups at the $40 \%$ and $50 \%$ criterion levels, but at no lesser criterion. This pattern of results is important because it indicates not only that Group O-S was slower than Group S-O in achieving a relatively large discrimination difference, but also that the rates of development of this achievement did not differentially fluctuate for the groups over trials. A similar analysis for Phase 2 revealed no significant difference at any discrimination criterion.

\section{Visible Urine}

An attempt was made to assess the importance of visible donor urine for the discrimination of $\mathrm{R}$ and $\mathrm{N}$ odors. Since deposition of urine has been implicated as a prominent marking device in rodents, it is possible that it might have been differentially present on $\mathrm{R}$ and $\mathrm{N}$ trials. Frequency of urination was determined by visual observation of the goalbox floor after each donor pair completed a trial and before the test subject was given its trial. Based on the data of all donor pairs in both 
Table 1

Mean Number of Days for Groups S-O and O-S Needed for a Subject's Daily Reward-Nonreward Speed Difference to Exceed a Percentage of Reward-Nonreward Difference for All Subjects on the Last Day of the Phase

\begin{tabular}{rlllll}
\hline \multirow{2}{*}{$\begin{array}{c}\text { Percen- } \\
\text { tage }\end{array}$} & \multicolumn{2}{c}{ Phase 1 } & & \multicolumn{2}{c}{ Phase 2 } \\
\cline { 2 - 3 } \cline { 5 - 6 } & S-O & O-S & & S-O & O-S \\
\hline 5 & 3.167 & 2.83 & & 3.00 & 3.67 \\
10 & 3.167 & 2.83 & & 3.00 & 3.83 \\
20 & 3.667 & 5.33 & & 3.33 & 4.00 \\
30 & 4.830 & 5.33 & & 3.83 & 4.00 \\
40 & $4.830^{*}$ & $7.33^{*}$ & & 3.83 & 4.00 \\
50 & $5.000^{* *}$ & $8.00^{* *} \dagger$ & 5.33 & 4.67 \\
\hline
\end{tabular}

*Difference between Groups $S-O$ and $O-S$ was significant $(t(10)=$ $2.27, p<.05] . \quad$ * Difference between Groups $S-O$ and $O-S$ was significant $[t(10)=2.48, p<.05]$. TOne test subject in this group did not reach criterion and was assigned a score of 12 days.

phases of the study, urine was observed on $15.6 \%$ of the $\mathrm{R}$ trials and $12.9 \%$ of the $\mathrm{N}$ trials. A Wilcoxon test for two matched samples revealed no significant differences between $\mathrm{R}$ and $\mathrm{N}$ urine frequencies. These data would indicate that visible urine is probably not a controlling stimulus, although subtle quantities of urine not readily discriminable by ordinary observation may still occur. A similar conclusion was reached by McNeese and Ludvigson (Note 3 ) when they reported that fluorescent emissions, as an indicant of urine, were not detected on approximately $70 \%$ of all trials for a gonadectomized group that significantly discriminated $\mathrm{R}$ and $\mathrm{Ngoal}$ events. However, more frequent and larger amounts of fluorescent emissions were found on $\mathrm{N}$ than $\mathrm{R}$ trials in a sham-operated group, which did not differ in discrimination from the gonadectomized group.

\section{DISCUSSION}

The present study investigated whether rats could utilize biological odors interchangeably to learn about reward contingencies. During Phase 1, rats in Group S-O discriminated conspecific odors of reward and nonreward in a manner consistent with previous work, running fast in the presence of $\mathrm{R}$ odor and slowly in response to $\mathrm{N}$ odor (e.g., Ludvigson \& Sytsma, 1967; Seago et al., 1970; Eslinger \& Ludvigson, Note 1). In this group, the conditioned approach tendencies were consonant with unlearned reactions to $\mathrm{R}$ and $\mathrm{N}$ odors (Collerain, 1978; Collerain \& Ludvigson, 1972,1977; Mellgren et al., 1973). In contrast, for Group O-S, an R goal event was signaled by conspecific $\mathrm{N}$ odor, and an $\mathrm{N}$ goal event was signaled by conspecific $\mathrm{R}$ odor. Although this stimulus situation required the reversal of any unlearned reactions, rats in Group O-S discriminated $\mathrm{R}$ and $\mathrm{N}$ goal events based upon the opposite odor cues by running fast in the presence of $\mathrm{N}$ odor and slowly to $\mathrm{R}$ odor. Thus, the imposition of a reward schedule opposite to that of donors did not prevent the development of discrimination. Similarly,
Similarly, in Phase 2, both groups, operating under schedules that were the reverse of those in Phase 1, were able to establish significant discrimination. Thus, all rats demonstrated the associative and response capabilities necessary to use $\mathrm{R}$ and $\mathrm{N}$ odors interchangeably as discriminative cues.

As anticipated, however, the opposite schedule did slightly retard the development of discrimination in Phase 1 relative to the same schedule. This retardation was temporary, though, and it was not evident in Phase 2. The fact that it was absent from Phase 2 suggests that the retardation was not the result of a kind of innate associative constraint or "impediment." The retardation seems more reasonably attributable to interfering unlearned reactions, avoidance of $\mathrm{N}$ odor and/or approach to $\mathrm{R}$ odor, which habituate with experience with the odor.

The results of this experiment relate to the larger issue of the nature and role of chemical signals in mammalian communication systems. Beauchamp, Doty, Moulton, and Mugford (1976) have argued cogently that chemical communication research at the mammalian level cannot receive much guidance from the work with insects that seeks to discover, isolate, and identify "pheromones" (i.e., chemically simple substances having a clear functional significance that evoke quite specific, stereotyped responses within a single species and so function by virtue of a large degree of genetic program. ming). That is, the idea that mammalian chemical signals function like externally secreted hormones in ways fundamentally different from other kinds of stimuli is untenable. The present work, which clearly shows that odors acquired significance for rats but had some unlearned, readily habituated significance at the outset of the study, would appear to be a case illustrative of part of their argument. Odors of $\mathrm{R}$ and $\mathrm{N}$ appear to be olfactory stimuli that are not constrained to signal only "prewired" responses, that is, approach to R odor and avoidance of $\mathbf{N}$ odor. Their adaptive significance exceeds this simple relationship. However, the results of this experiment do not rule out the possibility that $R$ and $\mathrm{N}$ odors may have limited utilization under certain other circumstances (Davis, Prytula, Noble, \& Mollenhour, 1976; Eslinger \& Travis-Neideffer, Note 4).

\section{REFERENCE NOTES}

1. Eslinger, P. J., \& Ludvigson, H. W. Discriminability of reward and frustration odors as a function of individual and sex differences in odor production. Paper presented at the meeting of the Southwestern Psychological Association, New Orleans, April 1978

2. Taylor, R., \& Ludvigson, H. W. Selective removal of runwav odors to pinpoint olfactory control of double alternation patterning in rats. Paper presented at the meeting of the Southwestern Psychological Association, Fort Worth, Texas, April 1977

3. McNeese, R. \& Ludvigson, H. W. Searching for the source of frustration odor. Paper presented at the meeting of the Southwestern Paychological Association, Houston. April 1975. 
4. Eslinger, P. J., \& Travis-Neideffer, M. N. Utilization of odor cues as a function of like or unlike motivational-reward operations in donor and test rats. Paper presented at the meeting of the Southwestern Psychological Association, San Antonio, Texas, April 1979.

\section{REFERENCES}

Beauchamp, G., Doty, R., Moulton, D., \& Mugford, R. The pheromone concept in mammalian communication. A critique. In $\mathbf{R}$. Doty (Ed.), Mammalian olfaction, reproductive processes, and behavior. New York: Academic Press, 1976.

Bolles, R. C. Species-specific defense reactions and avoidance learning. Psvchological Review, 1970, 71, 32-48.

Collerain, I. Frustration odor in rats receiving small numbers of prior reward running trials. Journal of Experimental Psychology: Animal Behavior Processes, 1978, 4, 120-130.

Collerain, I., \& Ludvigson, H. W. Aversion of conspecific odor of frustrative nonreward in rats. Psychonomic Science, 1972, 27, 54-56.

Collerain, I., \& Ludvigson, H. W. Hurdle-jump responding in the rat as a function of conspecific odor of reward and nonreward. Animal Learning \& Behavior, 1977, 5, 177-183.

Davis, S. F., Prytula, E. R., Noble, M. J., \& Mollenhour, M. N. Motivational specificity of the signal value of odor cues. Animal Learning \& Behavior, 1976, 4, 407-410.

Hinde, R., \& Stevenson-Hinde, J. (Eds.). Constraints on learning, limitations and predispositions. New York: Academic Press, 1973.
Ludvigson, H. W. Runway behavior of the rat as a function of intersubject reward contingencies and constancy of daily reward schedule. Psychonomic Science, 1969, 15, 41-43.

Ludvigson, H. W., McNeese, R. R., \& Collerain, I. Longterm reaction of the rat to conspecific (frustration) odor. Animal Learning \& Behavior, 1979, 7, 251-258.

Ludvigson, H. W., \& Srtsma, D. The sweet smell of success: Apparent double alternation in the rat. Psychonomic Science, $1967,9,283-284$.

Mellgren, R., Fouts, R., \& Martin, J. Approach and escape to conspecific odors of reward and nonreward in rats. Animal Learning \& Behavior, 1973, 1, 129-132.

Morrison, R. R., \& Ludvigson, H. W. Discrimination by rats of conspecific odors of reward and nonreward. Science, 1970, 167, 904-905.

Prytula, R. E., \& Davis, S. F. Runway performance as a function of positively and negatively correlated olfactory cues. Psychological Reports, 1974, 35, 735-740.

Prytula, R. E., \& Davis, S. F. The relationship between locus of odor cues and double alternation responding in the rat. Animal Learning \& Behavior, 1976, 4, 352-356.

Seago, J. D., Ludvigson, H. W., \& Remley, N. R. Effects of anosmia on apparent double alternation in the rat. Journal of Comparative and Physiological Psychology, 1970, 71, 435-442.

Seligman, M., \& HaGer, J. (Eds.). The biological boundaries of learning. New York: Appleton, 1972.

(Received for publication April 16, 1979; revision accepted April 14, 1980.) 